\title{
$n$-Back task performance and corresponding brain-activation patterns in women with restrictive and bulimic eating-disorder variants: Preliminary findings
}

\author{
Mimi Israel ${ }^{\mathrm{a}, \mathrm{b}, \mathrm{c}, *}$, Michael Klein $^{\mathrm{d}, \mathrm{e}}$, Jens Pruessner ${ }^{\mathrm{b}, \mathrm{c}}$, Lea Thaler ${ }^{\mathrm{a}, \mathrm{b}, \mathrm{c}}$, Michael Spilka ${ }^{\mathrm{a}, \mathrm{c}}$, \\ Simona Efanov ${ }^{\mathrm{a}, \mathrm{c}}$, Anne-Sophie Ouellette ${ }^{\mathrm{a}, \mathrm{c}}$, Marcelo Berlim ${ }^{\mathrm{a}, \mathrm{b}, \mathrm{c}}$, Nida Ali ${ }^{\mathrm{b}, \mathrm{c}}$, \\ Thomas Beaudry ${ }^{\mathrm{b}, \mathrm{c}}$, Frederique Van den Eynde ${ }^{\mathrm{a}, \mathrm{b}, \mathrm{c}}$, Claire-Dominique Walker ${ }^{\mathrm{b}, \mathrm{c}}$, \\ Howard Steiger ${ }^{\mathrm{a}, \mathrm{b}, \mathrm{c}}$ \\ a Eating Disorders Program, Douglas University Institute, Montreal, Quebec, Canada \\ ${ }^{\mathrm{b}}$ McGill University, Psychiatry Department, Montreal, Quebec, Canada \\ ${ }^{\mathrm{c}}$ Research Centre, Douglas University Institute, Montreal, Quebec, Canada \\ d McGill University, Department of Psychology, Montreal, Quebec, Canada \\ e Montreal Neurological Institute, Cognitive Neuroscience Unit, Montreal, Quebec, Canada
}

Keywords:

Eating disorders

Functional neuroimaging

Executive function

Anorexia

Bulimia

Binge eating

\begin{abstract}
A B S T R A C T
Eating disorder (ED) variants characterized by "binge-eating/purging" symptoms differ from "restrictingonly" variants along diverse clinical dimensions, but few studies have compared people with these different eating-disorder phenotypes on measures of neurocognitive function and brain activation. We tested the performances of 19 women with "restricting-only" eating syndromes and 27 with "bingeeating/purging" variants on a modified $n$-back task, and used functional magnetic resonance imaging (fMRI) to examine task-induced brain activations in frontal regions of interest. When compared with "binge-eating/purging" participants, "restricting-only" participants showed superior performance. Furthermore, in an intermediate-demand condition, "binge-eating/purging" participants showed significantly less event-related activation than did "restricting-only" participants in a right posterior prefrontal region spanning Brodmann areas 6-8-a region that has been linked to planning of motor responses, working memory for sequential information, and management of uncertainty. Our findings suggest that working memory is poorer in eating-disordered individuals with binge-eating/purging behaviors than in those who solely restrict food intake, and that observed performance differences coincide with interpretable group-based activation differences in a frontal region thought to subserve planning and decision making.
\end{abstract}

\section{Introduction}

Most diagnostic criteria for the eating disorders (EDs) differentiate anorexia nervosa (AN) from bulimia nervosa (BN) (e.g., American Psychiatric Association, 2013)-AN being characterized by willful restriction of food intake and weight loss, BN by recurrent eating binges and compensations (through selfinduced vomiting, laxative misuse, or other means). As bingeeating and purging occur in many people with AN, the syndrome is further subdivided into "AN-restricting (AN-R)" and "AN-bingeeating/purging" (AN-BP) types, depending upon the presence or

\footnotetext{
* Corresponding author at: Eating Disorders Program, Douglas University Institute in Mental Health, 6605 LaSalle Blvd, Montreal, Quebec, Canada H4H 1R3. Tel.: +1 514761 6131x6141; fax: +1 5148884085 .

E-mail address: mimi.israel@douglas.mcgill.ca (M. Israel).
}

absence of binge-eating and purging behaviors. Individuals who binge and purge (whether they have AN-BP or BN) are noted to be more prone to behavioral disinhibition and affective instability than are those who solely restrict food intake (Rosval et al., 2006; Steiger et al., 2013; Wu et al., 2013), and various findings validate the concept that "restricting-only" and "binge-eating/purging" individuals, regardless of weight status, fall into separate classes (Keel et al., 2004; Williamson et al., 2005; Wade et al., 2006; Wildes and Marcus, 2013). The present study sought to evaluate the extent to which the "restricter-only" versus "binger-purger" distinction coincided with distinct neurocognitive tendencies.

\subsection{EDs, executive functions, and brain activations}

Executive functions are higher order cognitive processes that regulate such mental operations as selective attention, working 
memory, and inhibitory control (Elliott, 2003). Executive functions are thought to implicate "frontostriatal" neurocircuits encompassing the dorsolateral prefrontal cortex (DLPFC), the anterior cingulate cortex, and connected brain structures-including the premotor and motor cortices, dorsal cingulate cortex, frontal poles, and medial posterior parietal cortex (Phillips et al., 2003). Some functional neuroimaging studies in ED populations point to the presence of frontostriatal abnormalities and associated executivecontrol problems (Pietrini et al., 2011; Friederich et al., 2013). For instance, Marsh and colleagues reported that adults with BN responded more impulsively and made more errors than did healthy adults on a task necessitating the inhibition of responses that were "primed" by incongruent stimulus information. Furthermore, when responding correctly to incongruent trials, people with BN failed to activate frontostriatal circuits to the extent seen in healthy controls (Marsh et al., 2009). Severity of bulimic symptoms was inversely correlated with the extent of frontal activations. A follow-up study in adolescents with BN roughly replicated the imaging findings, but not group-based performance differences, or the association between activations and eating symptoms (Marsh et al., 2011). Also implicating frontostriatal circuitry, but showing different activation patterns, a second study in adolescents found those with AN-BP or BN, when performing a go/no-go task tapping inhibitory control, to show significantly greater activations in the hypothalamus and right DLPFC than did healthy adolescents or adolescents with AN-R, and greater activations than did normal controls in the bilateral precentral gyri, anterior cingulate cortex, and middle and superior temporal gyri (Lock et al., 2011). Eating-symptom severity was not correlated with extent of activations.

\subsubsection{The n-back task}

$n$-Back tasks tap people's ability to maintain attentional control while manipulating task-relevant information in working memory (Owen et al., 2005; Kobel et al., 2009). Generally, $n$-back tests have participants respond to a target stimulus in accordance with a changing rule: "zero-back" conditions ask for a response to a target stimulus in the current trial, "1-back" to the stimulus' occurrence in the previous trial (i.e., one trial back), "2-back" to its occurrence two trials back, and so on. The $n$-back is presumed to primarily measure functionality of the prefrontal cortex (PFC) and connected structures-including the lateral premotor cortex, dorsal cingulate and medial premotor cortex, dorsolateral and ventrolateral $\mathrm{PFC}$, frontal poles, and medial and lateral posterior parietal cortex (Owen et al., 2005; Rottschy et al., 2012). Some studies link working memory deficits with self-reported impulsiveness (Cheung et al., 2004; Stanford et al., 2009). Consistent with this notion, $n$-back performance is reported to be poor in people with borderline personality disorder (BPD: Hagenhoff et al., 2013), suicidality (Keilp et al., 2013), and attention-deficit/hyperactivity disorder (ADHD: McCarthy et al., 2014). When performing $n$-back tasks, ADHD patients reportedly activate bilateral middle frontal, cerebellar, occipital and parietal areas less than do controls (Valera et al., 2005; Bayerl et al., 2010). Likewise, on various tasks that recruit executive functions, BPD patients display hypoactivation of frontal areas-including the DLPFC (Krause-Utz et al., 2014). Additional findings suggest that acute stress hinders $n$-back performance, and dampens task-induced PFC (Porcelli and Delgado, 2009; Gärtner et al., 2014) and DLPFC (Qin et al., 2012) activations. Related to this point, emotional distraction has been observed to decrease working-memory task-induced DLPFC activation in BPD patients, but not in healthy individuals (Krause-Utz et al., 2012).

In ED populations, controlled studies have shown people with AN to display intact (Castro-Fornieles et al., 2010; Nikendei et al., 2011;
Lao-Kaim et al., 2014) or even supra-normal n-back performance (Dickson et al., 2008; Brooks et al., 2012). Two studies have examined brain activations during $n$-back performance in participants with AN (although neither compared AN-R with AN-BP types): the first found adolescents with active AN to show greater task-induced activation in temporal and parietal areas than did controls (Castro-Fornieles et al., 2010). The second noted no activation differences between adults with or without AN (Lao-Kaim et al., 2014). No study, to date, has studied $n$-back performance in $\mathrm{BN}$.

\subsection{The present study}

We compared $n$-back performance and corresponding brain activations between groups of "restricter-only" and "binger-purger" adults, using an $n$-back variant designed to tap performance across a range of working memory loads and stress conditions. The $n$-back in question, developed and used by a member of our group (J.P.), amalgamated standard $n$-back features tapping working memory (e.g., Owen et al., 2005) with stress-inducing features (see Dedovic et al. (2005)). The task was designed to push participants to their working memory limits, while incrementally adding task-induced stress. We reasoned that the challenge of maintaining executive controls under conditions of high cognitive load and stress might simulate real-life conditions conducive to dysregulation in susceptible individuals. Based on observations indicating good $n$-back performance in people with AN (Dickson et al., 2008; Brooks et al., 2012), and associating impulsivity with weaker working memory performance (Cheung et al., 2004; Stanford et al., 2009), we expected ED-R participants to show superior $n$-back performance to that of ED-BP participants. Of additional interest was to determine whether any observed performance differences coincided with PFC activation differences.

Given limited pilot funding available, we included no healthy comparison group, opting instead to optimize power to detect hypothetical differences between "restricter-only" and "bingeeating/purging" groups. We acknowledge that the lack of a healthy comparison group means that our study cannot localize findings relative to normative task performances and brain activations. Nonetheless, we felt that any observed "restricter"|“binger" differences as to neurocognitive function, especially if they corresponded to differences in brain activations, might help inform modeling of ED-subtype variations.

\section{Methods}

\subsection{Subjects}

Participants in this institutional ethics-board approved study were recruited through a specialized Eating Disorders Program. All provided informed consent, were right-handed according to the Edinburgh Handedness Inventory (Oldfield, 1971), and met DSM-IV-TR criteria (American Psychiatric Association, 2000) for an ED. Exclusion criteria were pregnancy, having a bipolar, psychotic, or active substance-use disorder, or having a history of seizures, brain trauma or neurological disease. Diagnoses, made using validated structured clinical interviews administered by trained and supervised interviewers, were corroborated by senior clinicians with years of experience with ED patients (M.I. and H.S.). Of 46 ED women recruited, 12 had AN/R, six AN/BP, 17 BN, seven a restrictive form of Eating Disorder Not Otherwise Specified (EDNOS) and four a bulimic variant of EDNOS. Based on the presence or absence of binge-eating and purging, we reclassified participants into a "restricter-only" (ED-R) group composed of 19 women (12 with AN-R and seven with a restricting form of EDNOS), and a "binge-eating/purging" (ED-BP) group, composed of 27 women (17 with BN, six with AN-BP and four with a bulimic EDNOS variant). All ED-BP participants displayed recurrent binge-eating and compensatory behaviors over the past 3 months; ED-R participants had shown no such signs over the past 3 months. Past history of eating symptoms, obtained using a retrospective Eating Disorders Examination (EDE) interview (Fairburn and Cooper, 1993), identified four ED-R participants to have had a history of bingeing and purging symptoms. Variance related to diagnostic crossover was handled statistically (see Section 3). We applied no similar correction for the opposite 
direction of crossover as a period of intense caloric restraint was invariably present in ED-BP participants. Psychoactive medication use was not exclusionary, and was being used by $50 \%$ of ED-R and $68 \%$ of ED-BP participants. Possible effects of medication use were controlled using covariance procedures (see Section 3). The proportion of members of each group using specific medication classes (sometimes more than one type) was as follows: antidepressants: $\mathrm{ED}-\mathrm{R}=47.4 \%$; $\mathrm{ED}-\mathrm{BP}=58.6 \%$; antipsychotics: $\quad \mathrm{ED}-\mathrm{R}=31.6 \% ; \quad \mathrm{ED}-\mathrm{BP}=20.7 \%$; anxiolytics: $\quad \mathrm{ED}-\mathrm{R}=0.0 \%$; $\mathrm{ED}-$ $\mathrm{BP}=13.8 \%$. Chi-squared tests detected no ED-R versus ED-BP differences on any proportion. Among ED-R participants, seven were taking a selective serotonin reuptake inhibitor (SSRI), two received venlafaxine, and six were prescribed an atypical antipsychotic. Among ED-BP participants, 16 were on an SSRI, six were on atypical antipsychotics, and two were on anxiolytics. Participants were on a stable medication regimen for at least 2 weeks before testing.

\subsection{Measures}

ED symptoms were measured using the Eating Disorders Examination (EDE: Fairburn and Cooper, 1993) interview, which reflects presence and severity of criterion ED symptoms (Cronbach alpha coefficients ranging from 0.67 to 0.90 ), and the Eating Disorders Inventory-3 (Garner, 2004), which assesses ED-specific and associated psychopathological symptoms ( $\alpha>0.80$ on all subscales). To tap comorbid symptoms, we administered the Barratt Impulsiveness Scale, version 11 (BIS: Patton et al., 1995), the Structured Clinical Interview for DSM-IV Axis I (SCID-I: First et al., 1996b) and the Structured Clinical Interview for DSM-IV Axis II (SCID-II, version 2: First et al., 1996a). On the BIS, $\alpha$ is 0.83 . SCID-I inter-rater reliability for ED participants ranges from 0.57 to 1.00 (Grilo et al., 2004). SCID-II kappa values range from 0.40 to 0.98 for categorical diagnoses; internal consistency coefficients range from 0.39 to 1.0 (Zanarini et al., 2000).

\subsection{Neuropsychological task}

We used a computerized $n$-back variant that coupled the working memory component definitive of all $n$-back tasks (e.g., Owen et al., 2005) with a stressinduction component inspired by Dedovic et al. (2005). The task required participants to respond to target digits presented directly on a screen (e.g., the digit 7) or indirectly as the solution to an arithmetic problem (e.g., $5+2=7$ ) according to a variable $n$-back rule requiring a response to the digit presented in the current trial (0-back), to the digit's occurrence in the previous trial (1-back), or to the digit's occurrence two trials back (2-back). Other features of the task were designed to heighten performance pressure: Feedback ("correct" or "incorrect") was displayed after each response. The $n$-back rule $(0,1$ or 2 trials back) was varied without warning. The duration of the timer window (default $=5 \mathrm{~s}$ ) was adapted to the participant's performance such that after two consecutive correct answers, the duration was decreased by $10 \%$, and after two consecutive errors, it was increased by $10 \%$. The $n$-back applied here can be obtained through one of the authors (J.P.).

Participants completed two runs of the task, each involving Rest, Control, and Experimental conditions. The Rest condition (two blocks of eight scanner acquisitions each) had participants view a neutral display without any targets. The Control condition (two blocks of 38 acquisitions each) had participants perform 0-back Math trials only, with a fixed default window. The Experimental conditions included all combinations of Digits and Math trials with the adaptive time-out window, and with two blocks of 50 acquisitions each. Testing occurred between 14.00 and $19.00 \mathrm{~h}$.

\subsection{Image acquisition}

Functional magnetic resonance imaging (fMRI) data were acquired on a 1.5 Siemens MAGNETOM Sonata. T2* weighted volumes with blood oxygenation leveldependent contrast were obtained for fMRI scanning using the "Mosaic 64" sequence, with 34 slices per volume acquired every $2.88 \mathrm{~s}$ during the scanning (echo time $=50 \mathrm{~ms}$ ). Scanning time and stimulus presentation were synchronized using a trigger signal sent from the scanner to the computer presenting the stimuli. A horizontal acquisition sequence was chosen with an alignment along the long axis of the hippocampus using an interleaved slice order. Volumes were acquired continuously for $10 \mathrm{~min}$ (resting state fMRI) and then following tasks. Participants viewed stimuli on a semitransparent screen via a head-coil-mounted mirror system, and responded via a mouse connected by optical cable to a computer Following the functional session, a high-resolution T1-weighted anatomical volume (1 $\mathrm{mm}$ isotropic resolution) was acquired.

\subsection{Data analyses}

ED-R and ED-BP groups were compared on descriptive and clinical variables using chi-squared or $t$-tests. Participants' $n$-back performance was measured as percent correct, incorrect or "timed out" (trials in which the response did not arrive before the window closed) responses-with indices computed separately for each level of Task Difficulty (TD: 0-back, 1-back or 2-back) and Condition (Digits or
Math). Reaction times (RTs) to achieve correct and incorrect trials were recorded. Variables were subjected to a mixed design analysis of variance (ANOVA), assessing effects of Group (G: ED-R versus ED-BP), TD, and G $\times$ TD interactions. Digits and Math conditions were analyzed separately.

Imaging data were processed using FMRI Expert Analysis Tool (FEAT) Version 6.00, part of FMRIB's Software Library (FSL, www.fmrib.ox.ac.uk/fsl). For first level analyses (single runs for individual subjects), we applied motion correction using the FSL Motion Correction program FMRIB's Linear Image Registration Tool (MCFLIRT; Jenkinson et al., 2002), slice-timing correction using Fourier-space time-series phase-shifting, non-brain removal using the BET brain extraction tool (Smith, 2002), spatial smoothing using a Gaussian kernel of full width at halfmaximum (FWHM) $8.0 \mathrm{~mm}$; grand-mean intensity normalization of the entire 4D dataset by a single multiplicative factor; high-pass temporal filtering (Gaussianweighted least-squares straight line fitting, with $\sigma=50.0 \mathrm{~s}$ ). Time-series statistical analysis was carried out using FMRIB's Improved Linear Model (FILM) with local autocorrelation correction (Woolrich et al., 2001). Registration was carried out first to high-resolution structural images and then to Montreal Neurological Institute (MNI) standard space images. Registration to high resolution structural and/or standard space images was carried out using FLIRT, FMRIB's linear image registration tool (Jenkinson and Smith, 2001; Jenkinson et al., 2002). As the local deformations permitted by a non-linear method are thought to provide superior registration of inter-individual differences than do linear methods, registration from high-resolution structural to standard space was further refined using FMRIB's nonlinear image registration tool (FNIRT) (Andersson et al., 2007a, 2007b). For second level analyses (fixed effects analysis of two runs for an individual subject), a fixed effects model was used by forcing the random effects variance to zero in FMRIB's Local Analysis of Mixed Effects (FLAME; Beckmann et al., 2003; Woolrich et al., 2004; Woolrich, 2008). Third level analyses (mixed effects over multiple subjects) were carried out using FLAME stage 1 (Beckmann et al., 2003; Woolrich et al., 2004; Woolrich, 2008). The third level models allowed for elective inclusion of covariates. For instance, in one such analysis, we included Body-Mass Index (BMI), Age and Education. Each covariate was demeaned, with Education input along a scale from 1 (no schooling) to 7 (graduate degree). Mean Age, BMI and Education were 23.0 years (S.D. 5.3, range 18-38), 19.6 (S.D. 3.3, range 15.2-27.5), and 3.8 (S.D. 1.1, range 2-7), respectively.

$Z$ (Gaussianised T/F) statistical images were thresholded using Gaussian Random Field (GRF)-theory-based maximum height thresholding with a corrected significance threshold of $p<0.05$ (Worsley, 2001). Cluster-based thresholding was determined by $z>2.3$ for voxels and a corrected significance threshold of $p<0.05$ for clusters. ED-R participants were compared to ED-BP using un-paired samples $t$-tests. Given interest in frontal (executive) functions, we made an a priori decision to consider only frontal gray matter (excluding the primary motor area), setting the boundary for our determination between Brodmann areas 4 and 6 , so as to consider everything anterior to and including Brodmann area 6 (specifically, areas 6, 8, 9, $10-16,24,25,32,33$, and 44-47). Contrasts always compared activations occurring during the 1-back or 2-back rule within each condition (Digits and Math) with that obtained during the corresponding 0-back condition, which contrasted brain activations across situations that were equal in all respects except for cognitive demands. (Contrasting an active $n$-back condition to the "Rest" condition, in which the participant passively viewed the presentation display without trials being presented, would have only contrasted brain-activity while problem solving to that occurring while being mentally inactive.)

\section{Results}

\subsection{Participant characteristics}

Compared with ED-R participants, ED-BP participants tended to be slightly older, have higher BMIs, and have longer durations of illness (see Table 1). Likewise, ED-BP participants displayed higher scores on body dissatisfaction, bulimia, and impulsivity measures, and more current and lifetime major depression, lifetime panic disorder, and BPD, than did ED-R participants. The groups did not differ as to use of psychoactive medications $\left[\chi^{2}\right.$ ( 1 d.f. $)=1.418$; $p=0.234]$.

\subsection{Task performance}

Across most conditions, significant TD effects were observed on percent correct and incorrect responses, and on corresponding RTs (see Table 2). Significant Group $\times$ TD interactions were obtained on Digits Percent Correct and Digits Percent Timed-out, and there were significant main G effects on Digits Percent Correct, Digits RT Correct, Math Percent Correct, Math RT Correct and Math RT 
Table 1

Clinical and demographic characteristics of participants with restrictive and binge-eating/purging variants of eating disorders. (Isolated missing values are indicated by different $n$ and d.f. in the table.)

\begin{tabular}{|c|c|c|c|c|c|c|c|}
\hline \multirow{2}{*}{$\begin{array}{l}\text { Groups } \\
\text { Variables }\end{array}$} & \multicolumn{2}{|c|}{ Restricting-only ( $n=19)$} & \multicolumn{2}{|c|}{ Binge-eating/purging $(n=27)$} & \multirow[t]{2}{*}{ Statistic } & \multirow[t]{2}{*}{ d.f. } & \multirow[t]{2}{*}{$p$} \\
\hline & Mean & S.D. & Mean & S.D. & & & \\
\hline Age & 20.47 & 4.03 & 24.81 & 5.38 & $t=2.98$ & 44 & 0.005 \\
\hline Body mass index $\left(\mathrm{kg} / \mathrm{m}^{2}\right)$ & 17.74 & 2.16 & 20.90 & 3.32 & $t=3.64$ & 44 & 0.001 \\
\hline Duration of illness (years) & 5.14 & 4.97 & 9.25 & 5.25 & $t=2.67$ & 44 & 0.011 \\
\hline \multicolumn{8}{|l|}{ Frequency (per month/past 3 months) } \\
\hline Binge-eating & 0 & & 19.19 & 18.38 & - & & \\
\hline Purging & 0 & & 51.41 & 159.51 & - & & \\
\hline Weight (kg) & $(n=18)$ & & $(n=27)$ & & & & \\
\hline Highest & 57.59 & 8.29 & 65.27 & 12.13 & $t=-2.01$ & 43 & 0.051 \\
\hline Lowest & 36.18 & 5.23 & 43.70 & 7.66 & $t=-2.61$ & 43 & 0.012 \\
\hline Barratt Impulsiveness Scale & $(n=18)$ & & $(n=22)$ & & & & \\
\hline Total score & 60.53 & 7.35 & 75.67 & 9.58 & $t=-5.51$ & 38 & $<0.001$ \\
\hline Eating Disorders Inventory subscale scores & $(n=18)$ & & $(n=26)$ & & & & \\
\hline Drive for thinness & 11.44 & 6.74 & 14.83 & 5.28 & $t=-1.87$ & 42 & 0.069 \\
\hline Ineffectiveness & 8.89 & 8.16 & 12.92 & 7.30 & $t=-1.72$ & 42 & 0.093 \\
\hline Perfectionism & 9.22 & 4.40 & 8.85 & 3.53 & $t=0.30$ & 42 & 0.765 \\
\hline Interpersonal distrust & 5.61 & 5.47 & 6.11 & 4.16 & $t=-0.34$ & 42 & 0.733 \\
\hline Interoceptive awareness & 11.80 & 8.67 & 15.77 & 6.00 & $t=-1.68$ & 42 & 0.103 \\
\hline Maturity fears & 6.22 & 4.57 & 7.27 & 5.58 & $t=-0.66$ & 42 & 0.515 \\
\hline Ascetism & 7.79 & 5.37 & 7.65 & 2.70 & $t=0.10$ & 42 & 0.924 \\
\hline Impulse regulation & 5.28 & 4.63 & 7.41 & 6.12 & $t=-1.25$ & 42 & 0.219 \\
\hline Social insecurity & 7.00 & 5.57 & 8.31 & 5.21 & $t=-0.80$ & 42 & 0.430 \\
\hline Body dissatisfaction & 14.19 & 7.12 & 18.85 & 6.92 & $t=-2.64$ & 42 & 0.012 \\
\hline Bulimia & 0.75 & 1.13 & 9.28 & 4.68 & $t=-7.47$ & 42 & $<0.001$ \\
\hline \multirow[t]{2}{*}{ Comorbidities } & $(n=18)$ & & $(n=27)$ & & & & \\
\hline & Yes & No & Yes & No & & & \\
\hline \multicolumn{8}{|l|}{ Major depression } \\
\hline Current & 1 & 17 & 8 & 19 & $\chi^{2}=3.91$ & 1 & 0.048 \\
\hline Lifetime & 9 & 9 & 23 & 4 & $\chi^{2}=6.51$ & 1 & 0.011 \\
\hline \multicolumn{8}{|l|}{ Panic disorder } \\
\hline Lifetime & 0 & 18 & 5 & 22 & - & & \\
\hline Borderline personality disorder* & 0 & 19 & 6 & 21 & - & & \\
\hline
\end{tabular}

* Restricting-only: $n=19$.

Table 2

$n$-Back performance of participants with restrictive and binge-eating/purging variants of eating disorders.

\begin{tabular}{|c|c|c|c|c|c|c|c|c|c|}
\hline \multirow{3}{*}{$\begin{array}{l}\text { Level of difficulty } \\
\text { Variables }\end{array}$} & \multicolumn{6}{|c|}{ Groups, Mean and (S.D.) } & \multicolumn{3}{|c|}{ Repeated measures ANOVAS } \\
\hline & \multicolumn{3}{|c|}{ Restricting-only ( $n=19$ ) } & \multicolumn{3}{|c|}{ Binge-eating/purging $(n=27)$} & \multirow[t]{2}{*}{ Group } & \multirow[t]{2}{*}{ Difficulty } & \multirow[t]{2}{*}{ Group $\times$ Difficulty } \\
\hline & Oback & 1back & 2back & Oback & 1back & 2back & & & \\
\hline Digits \% correct & $83.28(12.19)$ & 77.49 (13.67) & $61.66(22.49)$ & $90.17(7.37)$ & $73.47(16.43)$ & $49.74(23.07)$ & $F(1,44) 0.81$ & $F(2,88) 46.52^{* * * * *}$ & $F(2,88) 4.20^{*}$ \\
\hline Digits \% incorrect & $3.61(3.07)$ & $12.81(13.70)$ & $28.06(23.10)$ & $4.50(4.84)$ & $20.56(14.77)$ & $40.15(20.92)$ & $F(1,44) 4.35^{*}$ & $F(2,88) 61.06^{* * *}$ & $F(2,88) 2.14$ \\
\hline Digits \% timeout & $13.12(11.01)$ & $9.70(8.86)$ & $10.28(11.42)$ & $5.33(6.40)$ & $5.96(7.50)$ & $10.11(11.29)$ & $F(1,44) 2.73$ & $F(2,88) 1.51$ & $F(2,88) 3.88^{*}$ \\
\hline Digits RT correct & $2.00(0.47)$ & $1.95(0.64)$ & $1.93(0.63)$ & $2.39(0.48)$ & $2.53(0.76)$ & $2.71(1.20)$ & $F(1,44) 8.04 \ldots$ & $F(2,88) 1.06$ & $F(2,88) 2.51$ \\
\hline Digits RT incorrect & $0.67(0.48)$ & $1.53(0.76)$ & $2.14(0.79)$ & $0.98(0.87)$ & $2.23(1.19)$ & $2.87(1.13)$ & $F(1,44) 9.34^{* * *}$ & $F(2,88) 45.40^{* * * * *}$ & $F(2,88) 0.84$ \\
\hline Math \% correct & $59.48(11.76)$ & $48.76(21.00)$ & $35.52(12.71)$ & $52.18(18.14)$ & $37.34(20.18)$ & $25.83(14.11)$ & $F(1,44) 5.72 *$ & $F(2,88) 42.58^{* * * \cdots * k}$ & $F(2,88) 0.29$ \\
\hline Math \% incorrect & $23.52(15.44)$ & $37.82(21.81)$ & $50.38(16.08)$ & $26.17(14.76)$ & 37.01 (16.87) & $46.83(20.75)$ & $F(1,44) 0.02$ & $F(2,88) 38.64^{* * * * * *}$ & $F(2,88) 0.66$ \\
\hline Math \% timeout & $17.00(12.20)$ & $13.42(10.70)$ & 14.10 (10.29) & 21.65 (15.47) & $25.66(18.82)$ & $27.34(20.88)$ & $F(1,44) 6.13^{*}$ & $F(2,88) 0.24$ & $F(2,88) 2.35$ \\
\hline Math RT correct & 4.43 (1.19) & $3.34(1.33)$ & $2.99(1.23)$ & $5.21(1.03)$ & $4.36(2.01)$ & $4.10(2.30)$ & $F(1,44) 8.14^{* * *}$ & $F(2,88) 9.43^{\text {****** }}$ & $F(2,88) 0.16$ \\
\hline Math RT incorrect & $4.56(2.20)$ & $4.41(1.56)$ & $3.90(1.38)$ & $5.98(1.83)$ & $4.95(1.71)$ & $4.72(1.70)$ & $F(1,44) 5.85^{*}$ & $F(2,88) 4.86^{* * *}$ & $F(2,88) 1.07$ \\
\hline
\end{tabular}

$$
\begin{aligned}
& * * * p=0.001 \\
& * * p<0.01 \\
& * p<0.05
\end{aligned}
$$

Incorrect. Results indicate ED-R participants' performance to be generally superior to that of ED-BP participants-reflected by more correct trials, fewer incorrect trials, and (in the more difficult Math condition) fewer Timeouts. Where interaction effects were obtained (Digits Percent Correct and Digits Percent Timeout), findings were consistent with superior performance by ED-R versus ED-BP participants at more demanding TDs.

\subsection{Brain activation patterns}

On three of four main contrasts that examined task-induced brain-activations (i.e., comparing 1-back Digits to 0-back Digits, and 1- and 2-back Math to 0-back Math), we obtained no groupbased differences. However, in the 2-back minus 0-back Digits contrast, a significant activation difference was observed, implying 


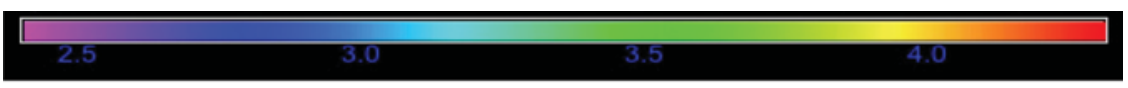

A

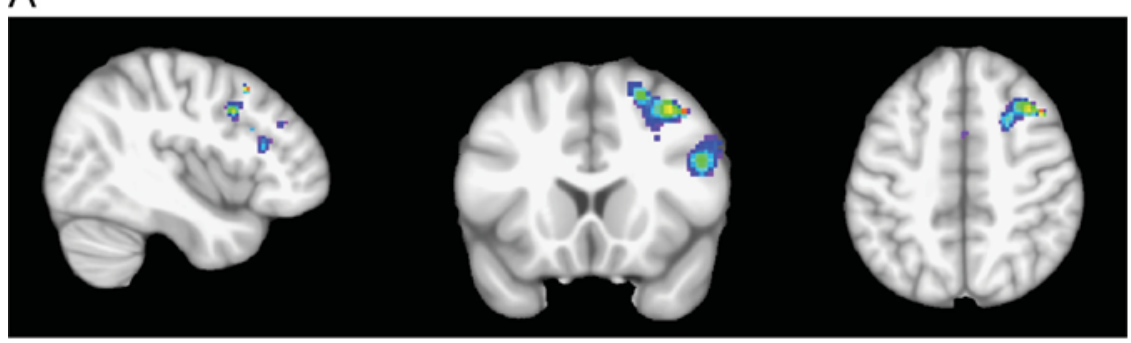

B

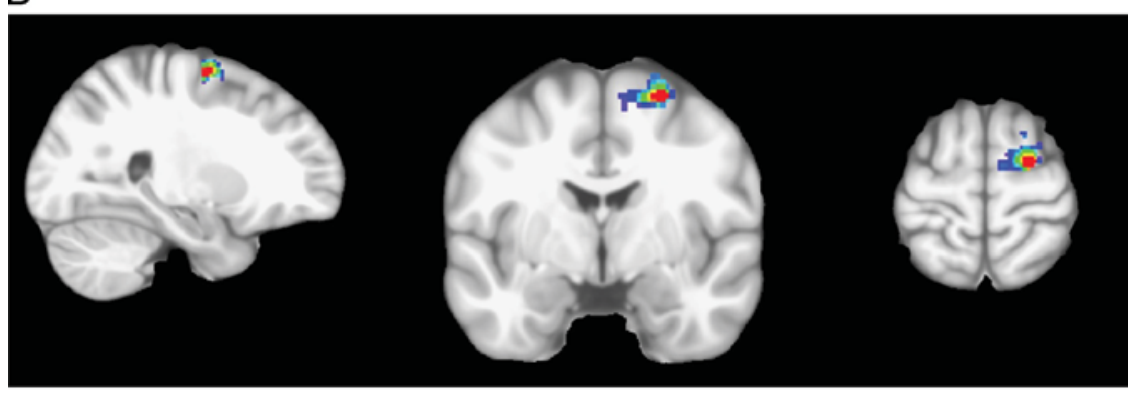

C

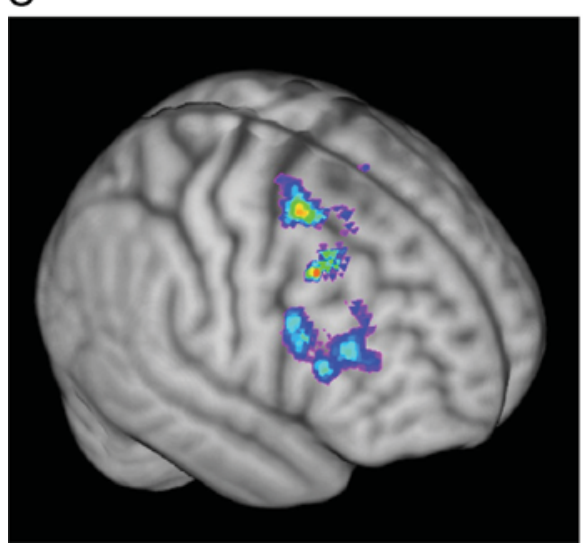

Fig. 1. Between-group activation differences in the 2-back Digits minus 0-back Digits contrast. Greater activation in women with eating disorders characterized by restriction (ED-R) compared to those who binge and/or purge (ED-BP) is noted in the right premotor cortex and the right superior frontal gyrus of the dorsolateral prefrontal cortex (Brodmann areas 6 and 8). (A) Prior to covariate adjustments, the cluster was significant using a family-wise error correction (FSL-generated cluster-based $p<0.006$ ), spanned 953 frontal lobe voxels and had a "center of gravity" at $X, Y, Z=+23.2,+9.58,+55.3$. A secondary and smaller neighboring cluster $(p<0.05$, right inferior frontal sulcus) spanned 593 voxels with a center of gravity at $X, Y, Z=+46.5,+22.7,+27$. Slice-wise images are viewed at MNI coordinates $X, Y, Z=+42,+18,+48$, which is the location of the peak voxel from the cluster $(t=4.1)$. The color plot represents the $t$-value at each voxel, and is the same for images $(\mathrm{a}-\mathrm{c})$. The images are displayed in neurological convention: right=right and left=left. (B) After adjusting for the covariates Age, BMI and Education, the cluster remained significant (FSL-generated clusterbased $p<0.04$ ), spanned 619 frontal lobe voxels and had a "center of gravity" at $X, Y, Z=+10.8,+3.4,+58.9$. Slice-wise images are viewed at MNI coordinates $X, Y, Z=+26$, $-4,+64$, which is the location of the peak voxel from the cluster $(t=6.0)$. (C) The same contrast from A, rendered in three dimensions in order to visualize the spatial extent of the significant clusters. (For interpretation of the references to color in this figure legend, the reader is referred to the web version of this article.)

greater average activation in the ED-R group in a right hemisphere cluster (involving 953 frontal lobe voxels and having a "center of gravity" at $x, y, z=+23.2,+9.58,+55.3)$, spanning a small anterior portion of Brodmann area (BA) 6 (implicating parts of the premotor cortex and, some authors would argue, DLPFC (Petrides, 2005; Badre and D'Esposito, 2009) to BA 8 (the right superior frontal gyrus, unambiguously implicating the DLPFC). The cluster was significant using a family-wise error correction (FSLgenerated cluster-based $p<0.006$ ). A smaller right DLPFC cluster (spanning 593 voxels with a center of gravity at $x, y, z=+46.5$, $+22.7,+27, p<0.05$ ) was located more laterally, primarily within the inferior frontal sulcus (see Fig. 1A). This cluster had an FSLgenerated cluster-based $p<0.05$. MNI coordinates were obtained from FSL outputs, and matched to Brodmann areas using the atlas provided by MRIcron (Rorden et al., 2007).
Adding Age, BMI and Education level as covariates, we obtained a significant activation difference (FSL-generated cluster-based $p<0.04$ ) centered in roughly the same location to that isolated in the preceding analysis, but encompassing a more-circumscribed region (see Fig. 1B). Reduced cluster size and statistical significance are both likely results of reduced statistical power, owing to introduction of covariates. In an ancillary analysis (performed separately to avoid over-parameterization of our model), we tested the 2-back Digits versus 0-back Digits contrast using psychoactive medication as a covariate, and found no substantive change as to significance of findings (FSL-generated cluster-based $p<0.03$ ). Similarly, in a separate analysis, we added Chronicity of Illness (in months) as a covariate and found comparable results to those of our first analysis (shown in Fig. 1A), with two significant clusters of activation (FSL-generated cluster-based $p<0.001$ and $p<0.05$ ) 
in essentially the same locations isolated by the main analysis. Furthermore, removal of four ED-R participants who had acknowledged a history of binge-eating or purging behavior did not substantively impact findings-which now showed a single larger cluster (FSL cluster-based $p<0.001$, spanning 2575 voxels, with a center of gravity at $x, y, z=+31.2,+14.6,+41.9$, in the region previously identified) and revealing differential brain activations between ED-R and ED-BP participants in the 2-back minus 0-back Digits contrast.

To account for possible effects of group differences as to $n$-back performance on neural activation, we tested the group activation difference obtained in the 2-back minus 0-back contrast, but applying "percent correct responses" in the 2-back condition as a covariate. The result showed a single cluster (FSL cluster-based $p<0.001$, spanning 1947 voxels, with a center of gravity at $x, y$, $z=+32.5,+14.8,+42.5)$. In other words, the effect again largely implicated the DLPFC. As a further test, we repeated our analyses identifying group-based differences in the DLPFC region of interest, but using correct trials only. Here, significant results were not obtained. Reduced power, associated with a reduced number of samplings within correct-trial groupings may, at least in part, explain the absence of significant effects.

Although our study's power for tests of relationships between severity of symptoms and brain activations was limited, we also examined the correlation between the cluster average activation (again in the BA 6 and BA 8 region of interest) during the 2-back minus 0-back Digits condition for each participant and each of the following values: EDE Total, Shape Concerns, Weight Concerns, Fear of Weight Gain, and Restriction scores, and Objective Binge Episodes, Vomiting Episodes (all evaluated over the preceding 28 days) and Barratt Impulsiveness total scores. None of the correlations achieved statistical significance.

\section{Discussion}

This study showed ED-R and ED-BP participants to perform differently on a specialized $n$-back task-with the ED-R group doing considerably better than the ED-BP group across most levels of task difficulty. In parallel, our findings showed a group-based difference as to brain-activations in one of the $n$-back conditions (the 2-back minus 0-back Digits contrast)-indicating the ED-R group to show more activation relative to the ED-BP group in a region encompassing the right DLPFC (BA 8) and a small portion of premotor cortex (BA 6) which, arguably, is functionally a component of the DLPFC. Anterior BA 6 has been considered to have "prefrontal" functioning and included with BA 8 as a part of the posterior-DLPFC (Petrides, 2005; Badre and D'Esposito, 2009). We note that the activation effect persisted after controls for effects of Age, BMI, Education, Illness Chronicity and psychoactive medication use.

$n$-Back tasks are believed to activate a frontostriatal circuit that includes the DLPFC (see Owen et al. (2005)). We are therefore encouraged to interpret the differential $n$-back performances observed in our ED-R and ED-BP groups, and the group-based difference in brain activation seen in one of our contrasts (mainly indicative of lesser PFC activation in the ED-BP group), as reflecting a group-based difference in neurocognitive functioning. The tendencies observed-indicating weaker working memory performance in ED-BP versus ED-R participants and (possibly) a corresponding propensity towards weaker task-induced activation in the DLPFC-would be compatible with group-based differences in clinical phenomenology, which associate the "binger-purger" profile with greater attentional and behavioral impulsivity than found in the "restricter-only" profile.
Because our study lacked a healthy comparison group, we cannot localize findings obtained in our ED-R and ED-BP groups in relation to normative results. Our study does, however, provide preliminary evidence (sufficient to motivate further investigation) of differential performances of these groups on the specialized $n$-back task applied here, and of possible group-based differences as to frontal brain-activations-as might suggest that the different clinical phenomenologies of interest coincide with different neuropsychiatric propensities.

Although we observed performance differences between people with "restrictive" or "binger-purger" ED variants across most of our $n$-back conditions, we detected activation differences in only the 2 -back versus 0 -back Digits contrast. We cannot ascertain why an activation difference would be observed in this contrast alone. However, we consider a "difficulty-threshold" type effectin which the difficulty of the 2-back Digits condition may have been calibrated to pick up a group-based activation difference that did not emerge when the task was easy for individuals in both groups (e.g., 1-back Digits or Math), or when individuals in both groups were pressed to or beyond their performance limits (as in the 2-back Math).

We add a comment concerning directionality of the groupbased activation effect observed. Some findings have actually associated stronger performance on working memory tasks with lesser prefrontal activations (e.g., Caldú et al., 2007; Dumontheil et al., 2011), not greater ones, as is the case in our findings. Inverse associations between performance and strength of brain activation have sometimes been interpreted to imply more efficient functioning of the relevant brain region. We note, however, findings that have linked memory load (Jaeggi et al., 2003) and motivation (Satterthwaite et al., 2012) positively with $n$-back induced activations in various brain structures. Following from the preceding, we assume that a positive association between brain activation and task performance observed here may be attributable to a particularly heavy load placed upon working memory and motivation by our $n$-back task-in response to which "restricter-only" participants may have been relatively more able than ED-BP participants to recruit needed cortical resources. Alternatively, we consider that ED-R participants, as reflected in their capacity to maintain relentless dietary restraint implies, may be capable of uniquely high degrees of motivation to realize goals. If so, the activation discrepancy we observe may, in part, reflect higher motivation to perform well in our ED-R participants.

We add that careful attention to differences associated with task parameters and sampling characteristics is warranted when attempting to interpret results obtained across studies in the ED literature. In this literature, some studies applying tasks presumed to tap frontal inhibitory controls show relatively weaker activations in frontal regions when comparing individuals displaying bulimic symptomatology to healthy control groups (Marsh et al., 2009; Joos et al., 2011), whereas others show relatively stronger activations (e.g., Lock et al., 2011). We suspect that apparent discrepancies may reflect differential brain-activation patterns associated with such factors as age, illness chronicity, comorbid traits, and variable task demands.

\subsection{Limitations}

This was an uncontrolled pilot investigation, designed to make a preliminary examination of hypothetical differences between "restricter-only" and "binger/purger" patients. Given the absence of a healthy control group, we can make no assertions about directional alterations in brain function in either clinical group studied. Furthermore, we applied FSL's recommended settings of cluster-based statistics (www.fmrib.ox.ac.uk/fsl), using a $Z$ threshold of 2.3 for voxels (which corresponds roughly to $p<0.01$ ) and a 
$p<0.05$ for clusters. While some authors have recommended application of a more conservative threshold in cluster-based analyses using Statistical Parametric Mapping (SPM: see Woo et al. (2014)), the preceding is not common practice, and runs the risk of unduly creating false negatives. Given an exploratory study examining group-based activation differences in what would predictably be a relatively broad region (namely the PFC and connected structures believed to be activated by $n$-back tasks), we have opted to apply a method that controls for multiple comparisons across a large brain region (i.e., FSL), instead of one that gives high spatial acuity at risk of false negatives (i.e., SPM). Nonetheless, readers should bear in mind that the localizations of functions suggested by our findings lack fine-grained spatial precision. Another limitation relates to the possible impact on our findings of psychiatric comorbidity. As results indicate, "binger-purger" participants were characterized by heightened comorbidity on various dimensions. The preceding raises the concern that group-based $n$-back performance differences are attributable to comorbidity instead of to the presence of bulimic symptoms. Moreover, we applied only partial controls for diagnostic crossover in the histories of our participants, and for medication use.

Not having obtained measures of IQ or ADHD, we were unable to control for effects of such factors upon task performance. We did, however, adjust for variance associated with educational level, and found no potential confound. Lastly, we note that the $n$-back variant we applied intentionally included the aspect of evaluating executive functioning under conditions of high performance demand and evaluative stress. While this manipulation was of theoretical interest, it risks confounding effects owing to stressreactivity with those purely associated with executive functions. In a related vein, findings that would validate the $n$-back variant applied here in healthy individuals have not yet been published, which limits our ability to comment on expected levels of performance in healthy populations.

\subsection{Implications for future research}

Future studies might build upon the present preliminary findings in several ways: addition of a normal-eater comparison group would help localize brain-activation findings in relation to normative levels, and a components analysis involving standard and modified $n$-back tasks might help separate effects due to demand upon executive functions from those attributable to stress or social-evaluative anxieties. In addition, a more thorough assay of comorbid psychopathological traits and tendencies, especially if obtained in samples large enough to support thorough analysis of sources of variance, would help tease apart effects that were attributable to categorical distinctions (such as that between restricter-only and binger-purger patients) from those owing to variations in trait correlates. Given evident heterogeneities that exist within categorical ED diagnoses, such efforts are necessary if we are to avoid misattribution of effects to ED pathology that might instead reflect concurrent trait tendencies.

\section{Conclusions}

This is the first study to document performance differences between "restricter-only" and "binger-purger" patients on a specialized $n$-back test, and to link group-based performance differences (in at least a preliminary fashion) to differential activations of DLPFC circuitry. Our findings make a case for the interpretation that different capacities for inhibitory control displayed by individuals suffering "restrictive" and "binge-purge" eating-disorder variants may implicate different average capacities to activate frontal brain regions underlying executive control. The suggested distinction would be quite compatible with a known association between deficits in working memory and propensity towards behavioral disinhibition (Bogg and Finn, 2010; Endres et al., 2011), given the tendency for individuals with "binge-purge" variants to display problems of inhibitory control, and those with "restrictive" ED variants to evince better task persistence. Most models of ED pathology assume that variations along an inhibition/disinhibition dimension account for differences seen between individuals who maintain relentless dietary restraint over prolonged periods (without succumbing to binge-eating/purging symptoms) and those who fall prey to binge-eating and purging following relatively modest dietary restraint. In this respect, we believe that our results, although preliminary, may add useful information to the effort to model neurocognitive and neuroanatomical correlates of different eating-disorder phenotypes. Furthermore, our findings contribute to the effort to root clinical tendencies seen in eating-disordered individuals to specific variations as to brain function which may, ultimately, be amenable to therapeutic intervention or serve as markers of risk, disorder activity, or recovery.

\section{Acknowledgments}

This research was supported by a grant from the Klarman Family Foundation for Research in Eating Disorders awarded to Howard Steiger.

\section{References}

American Psychiatric Association, 2000. Diagnostic and Statistical Manual of Mental Disorders (text revision (DSM-IV-TR). 4th ed. American Psychiatric Association Publishing, Inc., Washington, DC.

American Psychiatric Association, 2013. Diagnostic and Statistical Manual of Mental Disorders, 5th ed. American Psychiatric Association Publishing, Inc., Arlington, VA.

Andersson, J.L.R., Jenkinson, M., Smith, S.M., 2007a. Non-linear optimisation FMRIB technical report TR07JA1.

Andersson, J.L.R., Jenkinson, M., Smith, S.M., 2007b. Non-linear registration, aka spatial normalisation. FMRIB technical report TR07JA2.

Badre, D., D'Esposito, M., 2009. Is the rostro-caudal axis of the frontal lobe hierarchical? Nature Reviews Neuroscience 10, 659-669.

Bayerl, M., Dielentheis, T., Vucurevic, G., Gesierich, T., Vogel, F., Fehr, C., Stoeter, P. Huss, M., Konrad, A., 2010. Disturbed brain activation during a working memory task in drug-naive adult patients with ADHD. NeuroReport 21, 442-446.

Beckmann, C.F., Jenkinson, M., Smith, S.M., 2003. General multilevel linear modeling for group analysis in fMRI. NeuroImage 20, 1052-1063.

Bogg, T., Finn, P.R., 2010. A self-regulatory model of behavioral disinhibition in late adolescence: integrating personality traits, externalizing psychopathology, and cognitive capacity. Journal of Personality 78, 441-470.

Brooks, S.J., O’Daly, O.G., Uher, R., Schiöth, H.B., Treasure, J., Campbell, I.C., 2012 Subliminal food images compromise superior working memory performance in women with restricting anorexia nervosa. Consciousness and Cognition 21, $751-763$.

Caldú, X., Vendrell, P., Bartrés-Faz, D., Clemente, I., Bargalló, N., Jurado, M.Á., SerraGrabulosa, J.M., Junqué, C., 2007. Impact of the COMT Val108/158 Met and DAT genotypes on prefrontal function in healthy subjects. Neurolmage 37, 1437-1444.

Castro-Fornieles, J., Caldú, X., Andrés-Perpiñá, S., Lázaro, L., Bargalló, N., Falcón, C. Plana, M.T., Junqué, C., 2010. A cross-sectional and follow-up functional MRI study with a working memory task in adolescent anorexia nervosa. Neuropsychologia 48, 4111-4116.

Cheung, A.M., Mitsis, E.M., Halperin, J.M., 2004. The relationship of behavioral inhibition to executive functions in young adults. Journal of Clinical and Experimental Neuropsychology 26, 393-404.

Dedovic, K., Renwick, R., Mahani, N.K., Engert, V., Lupien, S.J., Pruessner, J.C., 2005 The Montreal imaging stress task: using functional imaging to investigate the effects of perceiving and processing psychosocial stress in the human brain. Journal of Psychiatry \& Neuroscience 30, 319-325.

Dickson, H., Brooks, S., Uher, R., Tchanturia, K., Treasure, J., Campbell, I.C., 2008. The inability to ignore: distractibility in women with restricting anorexia nervosa. Psychological Medicine 38, 1741-1748. 
Dumontheil, I., Roggeman, C., Ziermans, T., Peyrard-Janvid, M., Matsson, H., Kere, J., Klingberg, T., 2011. Influence of the COMT genotype on working memory and brain activity changes during development. Biological Psychiatry 70, 222-229.

Elliott, R., 2003. Executive functions and their disorders. British Medical Bulletin 65, $49-59$.

Endres, M.J., Rickert, M.E., Bogg, T., Lucas, J., Finn, P.R., 2011. Externalizing psychopathology and behavioral disinhibition: working memory mediates signal discriminability and reinforcement moderates response bias in approach-avoidance learning. Journal of Abnormal Psychology 120, 336-351.

Fairburn, C.G., Cooper, P., 1993. The eating disorders examination. In: Fairburn, C. Wilson, G.T. (Eds.), Binge Eating: Nature, Assessment and Treatment. Guilford, New York, pp. 317-360.

First, M.B., Gibbon, M., Spitzer, R.L., 1996a. Structured Clinical Interview for DSM-IV Axis-II Personality Disorders (SCID-II) (Version 2.0). Biometrics Research, New York State Psychiatric Institute, New York, NY.

First, M.B., Spitzer, R.L., Gibbon, M., Williams, J.B.W., 1996b. Structured Clinical Interview for DSM-IV Axis I Disorders-Patient Edition (SCID-I/P) (Version 2.0). Biometrics Research, New York State Psychiatric Institute, New York, NY

Friederich, H.-C., Wu, M., Simon, J.J., Herzog, W., 2013. Neurocircuit function in eating disorders. International Journal of Eating Disorders 46, 425-432.

Garner, D.M., 2004. The Eating Disorder Inventory-3: Professional Manual. Psychological Assessment Resources Inc., Odessa, FL.

Gärtner, M., Rohde-Liebenau, L., Grimm, S., Bajbouj, M., 2014. Working memoryrelated frontal theta activity is decreased under acute stress. Psychoneuroendocrinology 43, 105-113.

Grilo, C.M., Masheb, R.M., Lozano-Blanco, C., Barry, D.T., 2004. Reliability of the eating disorder examination in patients with binge eating disorder. International Journal of Eating Disorders 35, 80.

Hagenhoff, M., Franzen, N., Koppe, G., Baer, N., Scheibel, N., Sammer, G., Gallhofer, B., Lis, S., 2013. Executive functions in borderline personality disorder. Psychiatry Research 210, 224-231.

Jaeggi, S.M., Seewer, R., Nirkko, A.C., Eckstein, D., Schroth, G., Groner, R., Gutbrod, K., 2003. Does excessive memory load attenuate activation in the prefrontal cortex? Load-dependent processing in single and dual tasks: functional magnetic resonance imaging study. NeuroImage 19, 210-225.

Jenkinson, M., Bannister, P., Brady, M., Smith, S., 2002. Improved optimization for the robust and accurate linear registration and motion correction of brain images. Neurolmage 17, 825-841.

Jenkinson, M., Smith, S., 2001. A global optimisation method for robust affine registration of brain images. Medical Image Analysis 5, 143-156.

Joos, A.A.B., Saum, B., Zeeck, A., Perlov, E., Glauche, V., Hartmann, A., Freyer, T., Sandholz, A., Unterbrink, T., van Elst, L.T., Tüscher, O., 2011. Frontocingular dysfunction in bulimia nervosa when confronted with disease-specific stimuli. European Eating Disorders Review 19, 447-453.

Keel, P.K., Fichter, M., Quadflieg, N., Bulik, C.M., Baxter, M.G., Thornton, L., Halmi, K. A., Kaplan, A.S., Strober, M., Woodside, D.B., Crow, S.J., Mitchell, J.E., Rotondo, A., Mauri, M., Cassano, G., Treasure, J., Goldman, D., Berrettini, W.H., Kaye, W.H., 2004. Application of a latent class analysis to empirically define eating disorder phenotypes. Archives of General Psychiatry 61, 192-200.

Keilp, J.G., Gorlyn, M., Russell, M., Oquendo, M.A., Burke, A.K., Harkavy-Friedman, J., Mann, J.J., 2013. Neuropsychological function and suicidal behavior: attention control, memory and executive dysfunction in suicide attempt. Psychological Medicine 43, 539-551.

Kobel, M., Bechtel, N., Weber, P., Specht, K., Klarhöfer, M., Scheffler, K., Opwis, K., Penner, I.K., 2009. Effects of methylphenidate on working memory functioning in children with attention deficit/hyperactivity disorder. European Journal of Paediatric Neurology 13, 516-523.

Krause-Utz, A., Oei, N.Y.L., Niedtfeld, I., Bohus, M., Spinhoven, P., Schmahl, C., Elzinga, B.M., 2012. Influence of emotional distraction on working memory performance in borderline personality disorder. Psychological Medicine 42, 2181-2192.

Krause-Utz, A., Winter, D., Niedtfeld, I., Schmahl, C., 2014. The latest neuroimaging findings in borderline personality disorder. Current Psychiatry Reports 16, $1-13$.

Lao-Kaim, N.P., Giampietro, V.P., Williams, S.C., Simmons, A., Tchanturia, K., 2014. Functional MRI investigation of verbal working memory in adults with anorexia nervosa. European Psychiatry 29, 211-218.

Lock, J.M.D.P., Garrett, A.P., Beenhakker, J.M.S., Reiss, A.L.M.D., 2011. Aberrant brain activation during a response inhibition task in adolescent eating disorder subtypes. The American Journal of Psychiatry 168, 55-64.

Marsh, R., Horga, G., Wang, Z., Wang, P., Klahr, K.W., Berner, L.A., Walsh, B.T., Peterson, B.S., 2011. An fMRI study of self-regulatory control and conflict resolution in adolescents with bulimia nervosa. American Journal of Psychiatry 168 (11), 1210-1220

Marsh, R., Steinglass, J.E., Gerber, A.J., O’Leary, K.G., Wang, Z., Murphy, D., Walsh, T., Peterson, B.S., 2009. Deficient activity in the neural systems that mediate selfregulatory control in bulimia nervosa. Archives of General Psychiatry 66, 51-63.
McCarthy, H., Skokauskas, N., Frodl, T., 2014. Identifying a consistent pattern of neural function in attention deficit hyperactivity disorder: a meta-analysis. Psychological Medicine 44, 869-880.

Nikendei, C., Funiok, C., Pfüller, U., Zastrow, A., Aschenbrenner, S., Weisbrod, M., Herzog, W., Friederich, H.-C., 2011. Memory performance in acute and weightrestored anorexia nervosa patients. Psychological Medicine 41, 829-838.

Oldfield, R., 1971. The assessment and analysis of handedness: the Edinburgh inventory. Neuropsychologia 9, 97-113.

Owen, A.M., McMillan, K.M., Laird, A.R., Bullmore, E., 2005. N-back working memory paradigm: a meta-analysis of normative functional neuroimaging studies. Human Brain Mapping 25, 46-59.

Patton, J.H., Stanford, M.S., Barratt, E.S., 1995. Factor structure of the Barratt impulsiveness scale. Journal of Clinical Psychology 51, 768-774.

Petrides, M., 2005. Lateral prefrontal cortex: architectonic and functional organization. Philosophical Transactions of the Royal Society B: Biological Sciences 360, 781-795.

Phillips, M.L., Drevets, W.C., Rauch, S.L., Lane, R., 2003. Neurobiology of emotion perception I: the neural basis of normal emotion perception. Biological Psychiatry 54, 504-514.

Pietrini, F., Castellini, G., Ricca, V., Polito, C., Pupi, A., Faravelli, C., 2011. Functional neuroimaging in anorexia nervosa: a clinical approach. European Psychiatry 26, 176-182.

Porcelli, A.J., Delgado, M.R., 2009. Acute stress modulates risk taking in financial decision making. Psychological Science 20, 278-283.

Qin, S., Cousijn, H., Rijpkema, M., Luo, J., Franke, B., Hermans, E.J., Fernández, G., 2012. The effect of moderate acute psychological stress on working memoryrelated neural activity is modulated by a genetic variation in catecholaminergic function in humans. Frontiers in Integrative Neuroscience 6, 1-12.

Rorden, C., Karnath, H.-O., Bonilha, L., 2007. Improving lesion-symptom mapping. Journal of Cognitive Neuroscience 19, 1081-1088.

Rosval, L., Steiger, H., Bruce, K.R., Israel, M., Richardson, J., Aubut, M., 2006. Impulsivity in women with eating disorders: problem of response inhibition, planning, or attention? International Journal of Eating Disorders 39, 590-593.

Rottschy, C., Langner, R., Dogan, I., Reetz, K., Laird, A.R., Schulz, J.B., Fox, P.T., Eickhoff, S.B., 2012. Modelling neural correlates of working memory: a coordinate-based meta-analysis. NeuroImage 60, 830-846.

Satterthwaite, T.D., Ruparel, K., Loughead, J., Elliott, M.A., Gerraty, R.T., Calkins, M.E., Hakonarson, H., Gur, R.C., Gur, R.E., Wolf, D.H., 2012. Being right is its own reward: load and performance related ventral striatum activation to correct responses during a working memory task in youth. Neurolmage 61, 723-729.

Smith, S.M., 2002. Fast robust automated brain extraction. Human Brain Mapping $17,143-155$.

Stanford, M.S., Mathias, C.W., Dougherty, D.M., Lake, S.L., Anderson, N.E., Patton, J.H., 2009. Fifty years of the Barratt impulsiveness scale: an update and review. Personality and Individual Differences 47, 385-395.

Steiger, H., Bruce, K., Israël, M., 2013. Eating disorders. In: Widiger, T.A., Stricker, C. (Eds.), Comprehensive Handbook of Psychology: Clinical Psychology. John Wiley \& Sons, New York.

Valera, E.M., Faraone, S.V., Biederman, J., Poldrack, R.A., Seidman, L.J., 2005 Functional neuroanatomy of working memory in adults with attention-deficit/hyperactivity disorder. Biological Psychiatry 57, 439-447.

Wade, T.D., Crosby, R.D., Martin, N.G., 2006. Use of latent profile analysis to identify eating disorder phenotypes in an adult australian twin cohort. Archives of General Psychiatry 63, 1377-1384.

Wildes, J.E., Marcus, M.D., 2013. incorporating dimensions into the classification of eating disorders: three models and their implications for research and clinical practice. International Journal of Eating Disorders 46, 396-403.

Williamson, D.A., Gleaves, D.H., Stewart, T.M., 2005. Categorical versus dimensional models of eating disorders: an examination of the evidence. International Journal of Eating Disorders 37, 1-10.

Woo, C.W., Krishnan, A., Wager, T.D., 2014. Cluster-extent based thresholding in fMRI analyses: pitfalls and recommendations. Neurolmage 91, 412-419.

Woolrich, M., 2008. Robust group analysis using outlier inference. NeuroImage 41, 286-301.

Woolrich, M.W., Behrens, T.E.J., Beckmann, C.F., Jenkinson, M., Smith, S.M., 2004. Multilevel linear modelling for fMRI group analysis using Bayesian inference. Neurolmage 21, 1732-1747.

Woolrich, M.W., Ripley, B.D., Brady, M., Smith, S.M., 2001. Temporal autocorrelation in univariate linear modeling of fMRI data. Neurolmage 14, 1370-1386.

Worsley, K.J., 2001. Statistical analysis of activation images (Ch. 14). In: Jezzard, P., Matthews, P.M., Smith, S.M. (Eds.), Functional MRI: An Introduction to Methods. Oxford University Press, New York.

Wu, M., Hartmann, M., Skunde, M., Herzog, W., Friederich, H.C., 2013. Inhibitory control in bulimic-type eating disorders: a systematic review and metaanalysis. PLoS One 8, e83412.

Zanarini, M.C., Skodol, A.E., Bender, D., Dolan, R., Sanislow, C., Schaefer, E., Morey, L.C., Grilo, C.M., Shea, M.T., McGlashan, T.H., Gunderson, J.G., 2000. The collaborative longitudinal personality disorders study: reliability of axis I and II diagnoses. Journal of Personality Disorders 14, 291-299. 